\title{
Cryo-electron tomography of porcine reproductive and respiratory syndrome virus: organization of the nucleocapsid
}

\author{
Correspondence \\ Terje Dokland \\ dokland@uab.edu
}

Received 30 September 2008

Accepted 11 November 2008

\author{
Michael S. Spilman, ${ }^{1}$ Craig Welbon, ${ }^{2}$ Eric Nelson ${ }^{2}$ and Terje Dokland ${ }^{1}$ \\ ${ }^{1}$ Department of Microbiology, University of Alabama at Birmingham, Birmingham, AL 35294, USA \\ ${ }^{2}$ Department of Veterinary Science, South Dakota State University, Brookings, SD 57007, USA
}

Porcine reproductive and respiratory virus (PRRSV) is an enveloped positive-sense RNA virus of the family Arteriviridae that causes severe and persistent disease in pigs worldwide. The PRRSV virion consists of a lipid envelope that contains several envelope proteins surrounding a nucleocapsid core that encapsidates the RNA genome. To provide a better understanding of the structure and assembly of PRRSV, we have carried out cryo-electron microscopy and tomographic reconstruction of virions grown in MARC-145 cells. The virions are pleomorphic, round to egg-shaped particles with an average diameter of $58 \mathrm{~nm}$. The particles display a smooth outer surface with only a few protruding features, presumably corresponding to the envelope protein complexes. The virions contain a double-layered, hollow core with an average diameter of $39 \mathrm{~nm}$, which is separated from the envelope by a 2-3 nm gap. Analysis of the three-dimensional structure suggests that the core is composed of a double-layered chain of nucleocapsid proteins bundled into a hollow ball.

\section{INTRODUCTION}

Porcine reproductive and respiratory syndrome virus (PRRSV) is a widespread and highly infectious virus of pigs that causes a persistent and sometimes severe disease that is characterized by respiratory problems, weight loss and poor growth performance, as well as reproductive failure in pregnant sows (Rossow, 1998; Zimmerman et al., 1997). Measures to regulate the disease have been complicated by the pattern of persistent, subclinical infection with occasional epidemic outbreaks as well as the high heterogeneity of the virus (Batista et al., 2004; Blaha, 2000; Meng, 2000; Murtaugh et al., 2002).

PRRSV is a member of the family Arteriviridae of enveloped viruses with positive-sense (+) RNA genomes, that also includes lactate dehydrogenase-elevating virus (LDV) of mice, equine arteritis virus (EAV) and simian hemorrhagic fever virus (SHFV) (Plagemann, 1996). PRRSV is divided into two genotypes, the European, or type 1, also known as Lelystad virus, and American, or type 2 virus (Meng et al., 1995). There is considerable sequence variability within both groups and only about 50-60\% sequence identity between the two subtypes (Fang et al., 2007; Nelsen et al., 1999). Both subtypes now have a worldwide distribution.

Published online ahead of print on 3 December 2008 as DOI 10.1099/ vir.0.007674-0.

Videos of sections through selected tomograms are available with the online version of this paper.
Arteriviruses are grouped together with coronaviruses, toroviruses and roniviruses in the order Nidovirales (Cavanagh, 1997; Snijder et al., 1993). Like coronaviruses, the $15.4 \mathrm{~kb}$ PRRSV genome is expressed through a set of subgenomic mRNA transcripts, each used for the translation of one or two open reading frames (ORFs). ORF1a and ORF1b encode the non-structural proteins, including three proteases and the RNA-dependent RNA polymerase (Snijder \& Meulenberg, 1998); ORFs 2-5 encode glycosylated membrane proteins (GP2-GP5) and a small nonglycosylated protein E, ORF6 encodes a non-glycosylated membrane protein $(\mathrm{M})$, and ORF7 encodes the nucleocapsid $(\mathrm{N})$ protein that packages the viral RNA (Dea et al., 2000; Meulenberg et al., 1995). The major components of the envelope are GP5 and M, which form disulfide-linked heterodimers and are essential for particle assembly (Dea et al., 2000; Meulenberg et al., 1995; Wissink et al., 2005), while the minor proteins may play a role in defining tissue tropism (Wissink et al., 2004). All envelope proteins are required for infectivity (Wissink et al., 2005).

The 123-residue $\mathrm{N}$ protein is divided into an $\mathrm{N}$-terminal RNA-binding domain that is mostly disordered and positively charged (Yoo et al., 2003) and a C-terminal dimerization domain, comprising a four-stranded antiparallel $\beta$-sheet floor capped and flanked by $\alpha$-helices (Doan \& Dokland, 2003) (Fig. 1a, b). A predicted $\alpha$-helix $(\alpha 0)$ in the middle of the N-terminal region (PRRSV residues 25-35) may play a role in assembly, by analogy with a similar helix in the alphaviruses (Perera et al., 2001). $\mathrm{N}$ forms 
(a)
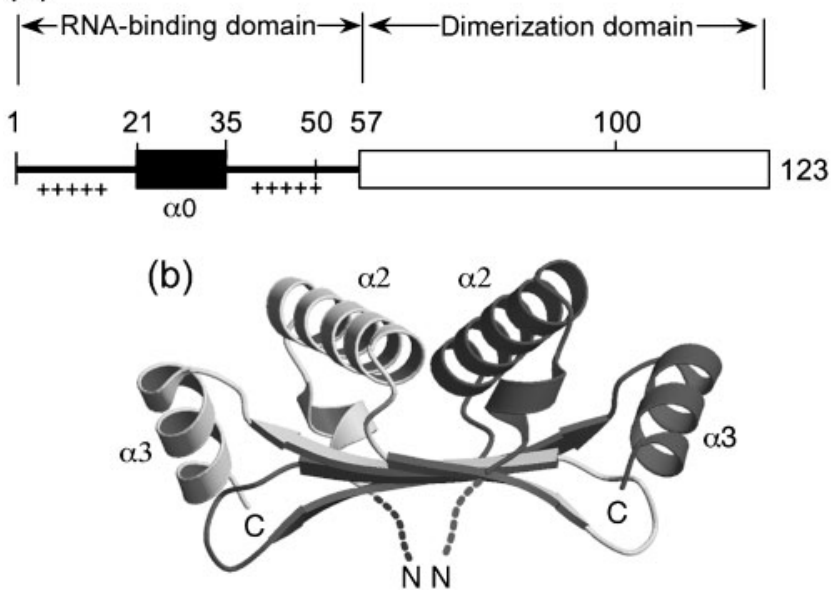

Fig. 1. The proteins of PRRSV. (a) Domain organization of the 123 amino acid $\mathrm{N}$ protein. The $\mathrm{C}$-terminal dimerization domain is shown as a white box, while the predicted $\alpha$-helix in the $\mathrm{N}$-terminal domain $(\alpha 0)$ is shown as a black box. Regions of positive charges are indicated by $(+)$ signs. (b) Ribbon representation of the crystal structure of the $\mathrm{N}$ dimer including residues 57-123 (PDB code 1P65) (Doan \& Dokland, 2003). The $\mathrm{N}$ - and C-termini are indicated, as are the $\alpha 2$ and $\alpha 3$ helices. The extension of the $\mathrm{N}$ termini into the RNA-binding domain is shown as stippled lines.

disulfide-linked homodimers via Cys 23 in this region (Wootton \& Yoo, 2003).

Very little is known about the structure of PRRSV virions and its proteins. Negative stain and thin section electron microscopy of PRRSV virions have shown a smooth, roughly spherical particle of about $60 \mathrm{~nm}$ diameter with a 20-30 nm diameter core; however, no internal structure could be discerned (Dea et al., 1995; Horzinek, 1981; Mardassi et al., 1994). We previously determined the crystal structure of the C-terminal domain of the $\mathrm{N}$ proteins of both PRRSV and EAV (Deshpande et al., 2007; Doan \& Dokland, 2003), but the organization of the structural proteins in the virion is still obscure. The purpose of the current study was to provide a better structural framework for understanding PRRSV assembly and pathogenesis. In this paper, we describe the structure of PRRSV virions based on cryo-electron microscopy (EM) analysis and tomographic reconstruction. We find that the virus has a pleomorphic morphology, a spherical to oval shape with a size ranging from about 50 to $65 \mathrm{~nm}$, a hollow, layered core of around $40 \mathrm{~nm}$ diameter and a smooth outer surface studded with a few envelope protein complexes. The overall organization of the virus is similar to that of coronaviruses like severe acute respiratory syndrome coronavirus (SARS-CoV; Neuman et al., 2006b), except for the absence of the prominent spikes characteristic of the coronaviruses. The structural analysis suggests that the PRRSV core consists of a helical nucleocapsid wrapped up into a hollow ball.

\section{METHODS}

Virus growth. MARC-145 monkey kidney cells were grown at $37{ }^{\circ} \mathrm{C}$ in Eagle's minimal essential medium (MEM) supplemented with $10 \%$ fetal bovine serum (Benfield et al., 1992). For preparation of high titre virus stocks, $180-240 \mathrm{ml}$ cells were infected with PRRSV strain SD23983 at an m.o.i. of $0.1 \mathrm{TCID}_{50}$ per cell and grown at $37^{\circ} \mathrm{C}$ in MEM supplemented with $2 \%$ equine serum for $36 \mathrm{~h}$, at which time only minimal cytopathogenic effect was observed. The cell supernatants were carefully harvested and clarified by centrifugation at $3000 \mathrm{~g}$ for $30 \mathrm{~min}$, followed by pelleting of the virus on a $0.5 \mathrm{M}$ sucrose cushion in TNC buffer (10 mM Tris, $100 \mathrm{mM} \mathrm{NaCl}, 2 \mathrm{mM} \mathrm{CaCl}_{2}, \mathrm{pH} 7.8$ ) at $80000 \mathrm{~g}$ for $90 \mathrm{~min}$ (Wu et al., 2001). The pelleted virus was resuspended in $2 \mathrm{ml}$ TNC or MEM and frozen at $-80{ }^{\circ} \mathrm{C}$ until microscopy was carried out. The frozen virus was then thawed, pelleted at $80000 \mathrm{~g}$ for $90 \mathrm{~min}$ and resuspended in $50 \mu \mathrm{l} 1 / 5 \mathrm{PBS}$ (2 $\mathrm{mM} \mathrm{Na}_{2} \mathrm{HPO}_{4}, 0.4 \mathrm{mM} \mathrm{KH} \mathrm{PO}_{4}, 30 \mathrm{mM} \mathrm{NaCl}, \mathrm{pH}$ 7.4). The virus was further dialysed into the same buffer on a $0.025 \mu \mathrm{m}$ filter for 45 min immediately prior to $\mathrm{EM}$ sample preparation. For $\mathrm{CsCl}$ gradient purification, the virus suspension was made to $1.32 \mathrm{~g} \mathrm{~cm}^{-3}$ $\mathrm{CsCl}$ and centrifuged for $23 \mathrm{~h}$ at 70000 r.p.m. in a Beckman NVT 90 rotor (Benfield et al., 1992).

Electron microscopy. For negative-stain EM, $2 \mu \mathrm{l}$ virus suspension was placed on glow-discharged 400 mesh carbon-only coated copper grids (Electron Microscopy Sciences) and stained with $1 \%$ uranyl acetate. For cryo-EM, $2 \mu$ virus suspension was placed on non-glow discharged 200 mesh Quantifoil holey carbon films (Quantifoil Micro Tools), blotted briefly (1-2 s) and frozen by plunging into liquid ethane, as previously described (Dokland \& $\mathrm{Ng}, 2006$ ). The grids were transferred to a Gatan 626 sample holder and observed at $-174{ }^{\circ} \mathrm{C}$ in an FEI Tecnai F20 electron microscope operated at $200 \mathrm{kV}$. Images were recorded on a Gatan Ultrascan $4 \mathrm{k} \times 4 \mathrm{k}$ CCD camera at a magnification of $\times 65500-81200$ with a defocus of 1.5-2.5 $\mu \mathrm{m}$ under low-dose conditions.

Tomographic data collection and reconstruction. For tomographic data collection, $6 \mathrm{~nm}$ BSA gold tracer (Electron Microscopy Sciences) was added to the sample to serve as fiducial markers during alignment. Tomographic data were acquired semi-automatically using the FEI Xplore3D software on the Tecnai F20 microscope operated at $200 \mathrm{kV}$. The images were collected at a magnification of $38000 \times$ and a nominal defocus of $-6 \mu \mathrm{m}$ with the CCD camera in binned mode, resulting in a pixel size of $7.9 \AA(0.79 \mathrm{~nm})$. Tilt series were collected over an angular range of $125^{\circ}$ to $135^{\circ}$ (typically from $-65^{\circ}$ to $+65^{\circ}$ ) with an angular step size of $1.0-2.0^{\circ}$, using either a linear or a Saxton scheme, resulting in 80-140 images per tilt series. Assuming a particle size of $600 \AA(60 \mathrm{~nm})$, the number of tilted views gives an upper resolution limit of $16 \AA(1.6 \mathrm{~nm})$, consistent with this choice of pixel size (Crowther et al., 1970). In practice, the poor signal-to-noise ratio limits the resolution to far lower. Due to the missing wedge of data at high angle, the resolution will be worse in the $z$ direction (parallel to the electron beam) than in the $x y$ plane. The total electron dose was $80-120$ electron $\AA^{-2}$ for the entire tilt series or about 1-2 electrons $\AA^{-2}$ per image (McEwen et al., 1995).

Tomographic data processing was done using IMOD (Kremer et al., 1996). After initial correlation alignment, fine alignment was done using the gold fiducial markers. The final reconstruction was calculated by the back projection algorithm after binning the images by an additional factor of 2, resulting in a pixel size of $15.8 \AA$ $(1.58 \mathrm{~nm})$. No phase correction was applied, since only data within the first zero of the CTF (around $40 \AA, 4 \mathrm{~nm}$ ) were used. The aligned frames were filtered with a $45 \AA(4.5 \mathrm{~nm})$ resolution two-dimensional Gaussian lowpass filter. The individual particle tomograms were filtered with a 3D bilinear filter with $\sigma 1=1$ and $\sigma 2=2 \times \mathrm{SD}$ of the map (Jiang et al., 2003) using the program proc3d in the EMAN suite 
(Ludtke et al., 1999). Map visualization and manipulation was done using the Chimera package (Pettersen et al., 2004).

\section{RESULTS AND DISCUSSION}

\section{Cryo-EM of PRRSV virions}

The PRRSV type 2 field isolate SD-23983 was chosen in this study for its ability to grow to high titres in cell culture (Feng et al., 2001). This virus grew on MARC-145 monkey kidney cells to titres of $10^{6}-10^{7}$ p.f.u. $\mathrm{ml}^{-1}$ cell culture supernatant in $48 \mathrm{~h}$ with minimal amount of cell lysis. The virus was concentrated from the supernatant to a titre of about $10^{8}-10^{9}$ p.f.u. $\mathrm{ml}^{-1}$ by two rounds of pelleting. In some cases, virus was also purified further on a $\mathrm{CsCl}$ gradient, but this step was omitted in virus used for cryo$\mathrm{EM}$, as the virus yield was too low.

By negative-stain EM, CsCl-purified virus appears as roughly spherical to somewhat oval particles with a mean diameter of about $55 \mathrm{~nm}$ (Fig. 2a). In some cases, the particles appear larger, due to collapse and flattening of the virus on the supporting substrate (not shown). The particles display a mostly featureless surface, but a few protrusions can be observed. No internal structures are discernible in intact particles because of the inability of the stain to penetrate through the lipid envelope. However, in partially ruptured particles, the lipid bilayer and the core inside can be discerned (Fig. 2a, inset).

The virions were subsequently imaged by cryo-EM, which eliminates the distortions and artefacts associated with negative staining (Dokland \& Ng, 2006). By cryo-EM, the virions appear as round or egg-shaped particles ranging in diameter from 50 to $74 \mathrm{~nm}$, with a median value of $54 \mathrm{~nm}$ and only few particles larger than $60 \mathrm{~nm}$ (Fig. 2b). This oval shape and pleomorphic nature was previously observed in pictures of thin-sectioned and negatively stained PRRSV and other arteriviruses (Brinton, 1994; Horzinek, 1981; Snijder \& Meulenberg, 1998), and suggest that PRRSV is not an icosahedrally ordered, isometric virus like e.g. flaviviruses and alphaviruses.

The particles display a very smooth outline with few protruding features. The lipid bilayer of the envelope is clearly discernible, and in some places, the membrane has a set of cross-striations with a spacing of about $4 \mathrm{~nm}$ (Fig. 2b, inset). These striations possibly correspond to the transmembrane (TM) domains of the envelope proteins, of which M and GP5 compose the majority (Mardassi et al., 1996; Meulenberg et al., 1995). M and GP5 have very small ectodomains of about 16 and 30 residues, respectively, which corresponds to a feature of approximately $2 \mathrm{~nm}$ in size. Thus, a smooth particle outline is expected. A few features protruding from the membrane surface by about $4 \mathrm{~nm}$ are also visible (Fig. 2b). These protrusions most likely stem from the more bulky, less abundant membrane proteins, such as GP2 (Wissink et al., 2005).

The virions contain an internal core with an average diameter of $39 \mathrm{~nm}$, which is separated from the envelope by a $2-3 \mathrm{~nm}$ gap. The size and shape of the core generally follow those of the envelope and thus display considerable variation. The core consists of a $10 \mathrm{~nm}$ thick layer of density surrounding a central, lower density area, suggesting that the core is hollow. Occasionally, particles with a rectangular core are found (Fig. 2b), suggesting that the core might be organized as a spool or solenoid, with the round cores representing end-on views and rectangular cores representing side views. However, this interpretation was not supported by the three-dimensional analysis (see below).

\section{Comparison with other nidoviruses}

Coronaviruses and arteriviruses are grouped together in the order Nidovirales based on similarities in genome
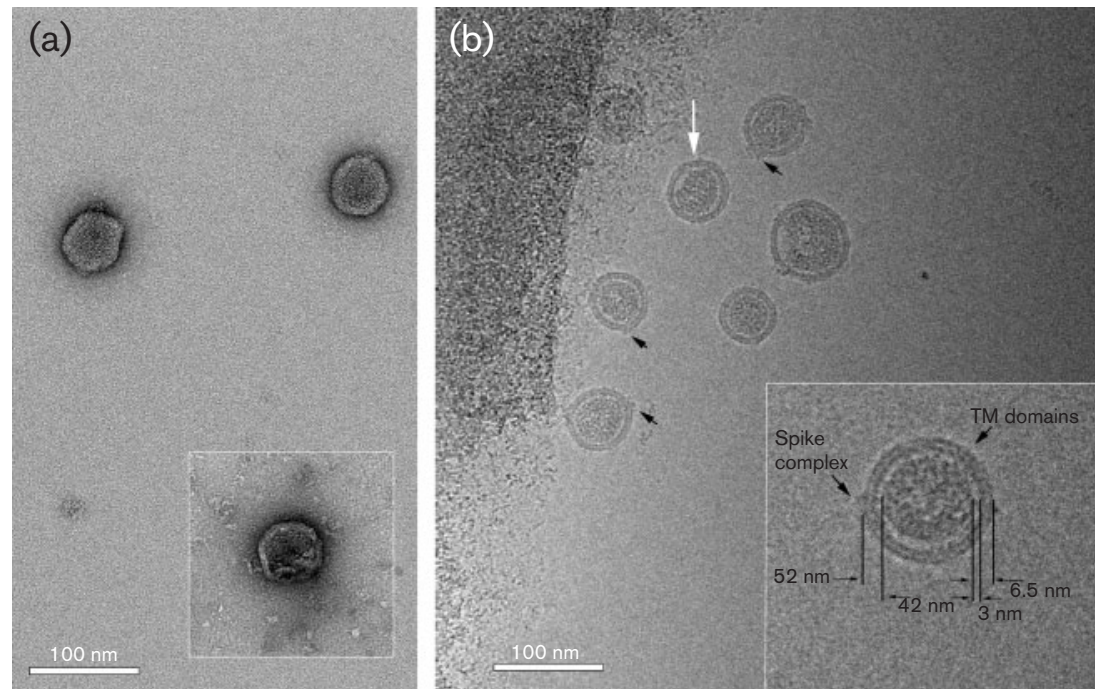

Fig. 2. Electron microscopy of PRRSV. (a) CsCl-purified PRRSV particles, negatively stained with uranyl acetate. The inset shows one partially disrupted particle, showing the lipid bilayer of the envelope. Bar, $100 \mathrm{~nm}$. (b) Cryo-EM of PRRSV particles in vitreous ice. The dark area on the left is part of the carbon support film. Bar, $100 \mathrm{~nm}$. The white arrow points to a particle with a rectangular core. Black arrows indicate protruding features thought to correspond to complexes of the minor envelope proteins. Inset, $\times 2$ magnified view of a single, typical PRRSV particle with dimensions indicated. A presumed envelope spike complex is indicated, as is the striated appearance most likely corresponding to transmembrane domains. 
organization and expression. Recent data have suggested that this relationship also extends to structural features: Xray crystallography and nuclear magnetic resonance (NMR) structures of the C-terminal dimerization domain of the nucleocapsid protein of the two coronaviruses infectious bronchitis virus (IBV) and SARS-CoV (Chang et al., 2005; Chen et al., 2007; Jayaram et al., 2006; Yu et al., 2006) have revealed a fold that is topologically equivalent to that from the arteriviruses PRRSV and EAV (Deshpande et al., 2007; Doan \& Dokland, 2003). In the coronaviruses, this $120-130$ residue domain is embedded within a much larger protein of 350-422 aa, compared with the 123 residues of the full-length PRRSV N protein.

Coronaviruses have generally been described as pleomorphic, roughly $100 \mathrm{~nm}$ diameter particles (Holmes \& Lai, 1996). Recently, cryo-EM of SARS-CoV (Neuman et al., 2006a, b) revealed oblong, membrane-bound particles about 50-100 $\mathrm{nm}$ wide and up to $150 \mathrm{~nm}$ long, studded by the club-shaped protruding envelope proteins that give the family its characteristic 'corona'. Coronaviruses are considered to have a helical nucleocapsid (Davies et al., 1981; Sturman et al., 1980). The exact organization of this nucleocapsid is not known, but some models have been proposed (Chen et al., 2007; Fan et al., 2005). The cryo-EM of SARS-CoV showed an amorphous core with the same elongated shape as that of the whole virion. Thus, the morphology of coronaviruses is very similar to what we observe here for PRRSV, except for the protruding spike proteins. These observations suggest that the relationship between corona- and arteriviruses extends to the supramolecular organization of the virion. Hence, it seems plausible that the arterivirus core may also be organized with the same helical arrangement of $\mathrm{N}$ protein as the coronaviruses.

\section{Tomographic reconstruction of PRRSV virions}

The heterogeneous and asymmetrical nature of the virus that is apparent in the electron micrographs means that reconstruction methods that involve either the application of symmetry or averaging between multiple particles are not suitable. Although it cannot be completely excluded a priori that a subset of individual particles have icosahedral symmetry, the generally irregular shape would indicate that such particles are very rare. Instead, the only way to generate three-dimensional information is by a general method like tomography, in which multiple views of the same particles are obtained by tilting the sample in the microscope. These views are then combined computationally into a 3D reconstructed volume (Frank, 2006; Lucic et al., 2005). The weakness of this technique is that only rather limited resolution is attainable, due to the lack of averaging, the high radiation sensitivity of frozen hydrated specimens, and the consequently poor signal-to-noise ratio of the reconstructions. Nevertheless, electron tomography has allowed structural information on asymmetrical viruses to be obtained at a resolution of up to $5 \mathrm{~nm}$ (Benjamin et al., 2005; Chang et al., 2007; Grunewald \& Cyrklaff, 2006; Harris et al., 2006).

A total of 15 tilt series were collected from two separate virus preparations, five of which were ultimately deemed of sufficiently high quality to calculate tomograms. Each tomogram contained on average 10-20 particles that were used for further analysis. A single $15.8 \AA(1.58 \mathrm{~nm})$ thick section through part of one complete tomogram is shown in Fig. 3. In this view, there are 11 normal-looking PRRSV virions with an average diameter of $56 \mathrm{~nm}$ and one abnormally large $76 \mathrm{~nm}$ particle. A large vesicle, possibly an exosome, presumably of cellular origin, and a smaller empty vesicle are also visible. While this view looks similar to the raw micrograph in Fig. 2(b), it is much clearer, since the information displayed in Fig. 3 results from only a single $15.8 \AA$ thick section, while the raw micrograph contains information superimposed through the entire volume. Some differences in size and appearance of the particles in this view are due to the fact that the position of each particle relative to the surface of the ice varies, and that the sectioning plane therefore does not pass through the centre of all particles. A video that shows serial sections through the whole tomogram can be found in Supplementary data S1, available in JGV Online.

Sub-tomograms containing one particle each were cropped out from the full tomograms and a spherical mask was applied to remove density arising from random noise, contaminating proteins and gold markers. Isosurface representations and central sections in the $z$ and $y$ directions (parallel and perpendicular to the electron beam, respectively) of a representative set of individual particle tomograms are shown in Fig. 4 (videos of sections in the $z$ and $y$ directions through two of these particles are shown in Supplementary data S2, available in JGV Online). The tomograms have a slightly drawn out appearance in the $z$ direction, most apparent in sections perpendicular to

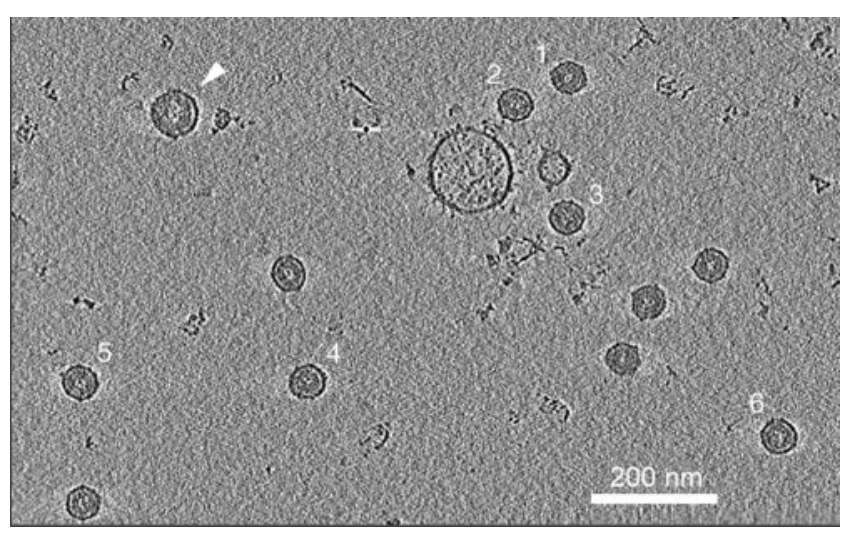

Fig. 3. Central section $[15.8 \AA$ thick $(1.58 \mathrm{~nm})]$ through part of a tomogram, showing several PRRSV particles. The arrowhead points to one abnormally large particle. The numbered particles correspond to those shown in more detail in Fig. 4. Bar, $200 \mathrm{~nm}$. 


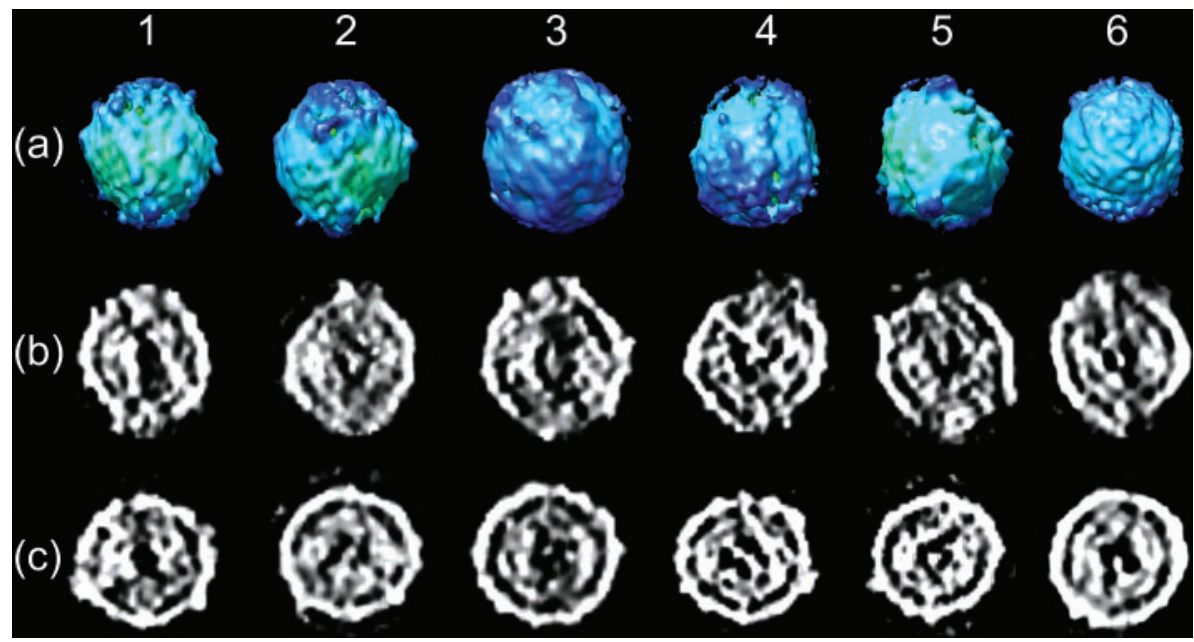

Fig. 4. Gallery of PRRSV virions. (a) Isosurface representations at $2 \sigma$ cut-off of representative particles picked from the tomogram. The numbering corresponds to the numbers in Fig. 3. The particles are viewed perpendicular to the $z$ axis, and colour-coded from red to blue according to distance from the centre of gravity. (b) Corresponding central sections through the tomograms in (a), perpendicular to the $z$ axis ( $y$ sections). Each image corresponds to two stacked sections [31.6 $\AA$ thickness $(3.16 \mathrm{~nm})$ ]. (c) Equatorial central sections parallel to the $z$ axis ( $z$ sections) through each of the particle tomograms in (a).

the $z$ axis (Fig. 4b), which is caused by the 'missing wedge' of information at high angles. Sections along the $z$ axis, however, are more or less centrosymmetrical (Fig. 4c).

The same features that were apparent in the micrographs are also obvious in the tomograms: the particles generally present a quite smooth and featureless outer surface (Fig. 4a). A few protruding features are evident on some of the particles, possibly corresponding to the bulkier envelope proteins, like GP2. At the resolution of the tomogram, the two leaflets of the lipid bilayer, separated by about $3 \mathrm{~nm}$, cannot be discerned, consistent with observations in other tomographic reconstructions of viruses (Harris et al., 2006; Wright et al., 2007).

\section{Organization of the nucleocapsid core}

The tomograms show that the viral core is not solid, but consists of a two-layered shell with a total thickness of about $10-11 \mathrm{~nm}$. This shell surrounds a hollow central cavity with a size that is related to the outer diameter of the particle, or about $13-14 \mathrm{~nm}$ in diameter for a normal, $56 \mathrm{~nm}$ particle. The core size and shape generally follow those of the envelope. Thus, abnormally large particles, such as the $76 \mathrm{~nm}$ particle in Fig. 3 (arrow), have large cores with a larger central cavity, but the same two-layered, $11 \mathrm{~nm}$ thick core shell. None of the cores had a rectangular outline, as would have been expected if the core was a spool or solenoid. Instead, sections through the tomograms in either direction $(x, y$ or $z$ ) generally reveal the same, more or less rounded outline of the core, after taking into consideration the stretching caused by the missing wedge (Fig. 4). Thus, the rectangular core seen in the micrograph in Fig. 2(b) is most likely a rare and abnormal occurrence.
The core is separated from the envelope by a $3 \mathrm{~nm}$ gap, traversed only by a few strands of density, suggesting that interactions between the core and the envelope proteins, if any, are weak and flexible.

In isosurface representation, the core looks disorganized and appears to consist of many strands of density bundled together into a ball (Fig. 5a). The tomograms typically have a single strong density in the centre of the particles, which is connected to the rest of the core only on one side (Fig. 5b). This density could conceivably correspond to one of the ends of the genome. The volume of the electron density enclosed by the core at a cut-off level of $2 \sigma$ above the mean corresponds to an average mass of $24.0 \pm$ $2.9 \mathrm{MDa}(25.5 \pm 3.0 \mathrm{MDa}$ at $1 \sigma$ cut-off $)$. If we subtract the mass of the genome (approx. $4753 \mathrm{kDa}$ ), this volume corresponds to 1415 copies of $\mathrm{N}$ in the core.

The $11 \mathrm{~nm}$ thick core shell comprises a series of elongated densities of approximately $7 \mathrm{~nm}$, organized into two discontinuous layers, in many cases giving the appearance of a chain with a link-to-link distance of about $10 \mathrm{~nm}$ (Figs 4, 5c). The elongated densities are roughly the same dimensions as an $\mathrm{N}$ protein dimer rendered at comparable resolution (Fig. 5c). However, the reconstruction also contains other density features that may be attributed to the RNA and the disordered N-terminal domain of N. This and the low resolution of the map prohibited a reliable and consistent fit of $\mathrm{N}$ into the electron density.

We previously suggested a model for the arterivirus core in which C-terminal domains of the $\mathrm{N}$ dimers formed a single-layered shell, while the RNA-binding N-terminal domains interacted with the RNA on the inside of the shell (Deshpande et al., 2007; Doan \& Dokland, 2003). The data 


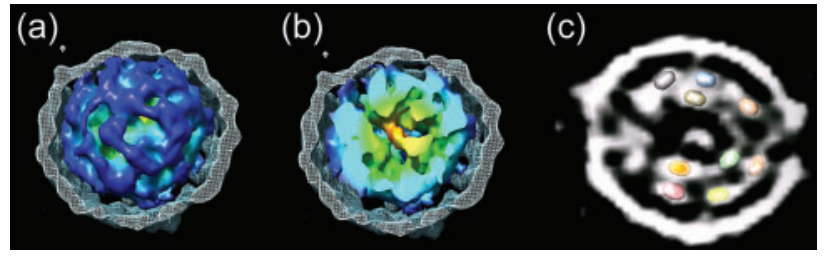

Fig. 5. Structure of the core. (a) Cutaway view of one PRRSV virion. The envelope, shown in mesh representation, was peeled away to reveal the internal core. The core is shown as an isosurface, coloured by the radius from the centre of the particle (from red to blue). In (b), the core has also been cut open to show the internal structure and in particular the characteristic central density (red-orange). (c) A $63 \mathrm{~nm}$ thick slab through the centre of one particle tomogram, with several copies of the crystal structure of the dimer of the $\mathrm{C}$-terminal domain of $\mathrm{N}$ rendered at a comparable resolution to the tomogram and superimposed on the oblong densities in the core.

presented here suggest an alternative model for the core, in which two layers of $\mathrm{N}$ dimers form a linked chain (Fig. 6). Since the C-terminal dimerization domain does not assemble on its own (Doan \& Dokland, 2003), it is likely that assembly involves interactions between the $\mathrm{N}$-terminal domains as well as the viral RNA. In our model, opposing $\mathrm{N}$ dimers interact via the $\mathrm{N}$-terminal domains, leading to the formation of the linked rings of density observed in the tomograms (Figs 4, 5). These interactions are likely to involve the predicted $\alpha 0$ helices between residues 21 and 35 (Fig. 1), by analogy with the $\alpha$-helix in the RNA binding domain of alphaviruses (Perera et al., 2001). The structure of the N-terminal domain is unknown, but if residues 2135 are assumed to be $\alpha$-helical while residues $36-57$ have a more extended conformation, it would span a distance of about 7-8 nm, sufficient to cover the observed distance between the two layers of density, while taking into account overlap between the opposing dimers (Fig. 6). In the core, the $\mathrm{N}$ proteins interact with the viral RNA via the positively charged residues in the $\mathrm{N}$-terminal domain, especially residues 35-51, causing the formation of a twisted ribbon or chain with the RNA in the centre. In the core, this chain is bundled into a roughly spherical shape, following the confines of the inside of the envelope and leaving a hollow interior. Overall, the core appears to be quite disorganized, and it was not possible to trace a consistent path of densities through the core.

This arrangement would not present a uniform surface for the envelope proteins to interact with, consistent with the observed gap between the core and the envelope and the failure to detect substantial interactions between $\mathrm{N}$ and the endodomains of M and GP5 in vitro (unpublished data). From a biological point of view, such an arrangement may be attractive as there is no need for disassembly of a closed shell-like core in order to release the genome into the cytoplasm after entry of the virus.

The conformation of the RNA in the nucleocapsid is unknown. In tobacco mosaic virus (TMV), the RNA describes a helical path corresponding to approximately $2.6 \mathrm{nt} \mathrm{nm}^{-1}$. Atomic force microscopy has shown that naked TMV RNA is more compact, at about $4.3 \mathrm{nt} \mathrm{nm}{ }^{-1}$ (Drygin et al., 1998; Kiselyova et al., 2001). Increased secondary structure formation would compact the RNA further. If we take $4.3 \mathrm{nt} \mathrm{nm}^{-1}$ as a reasonable average

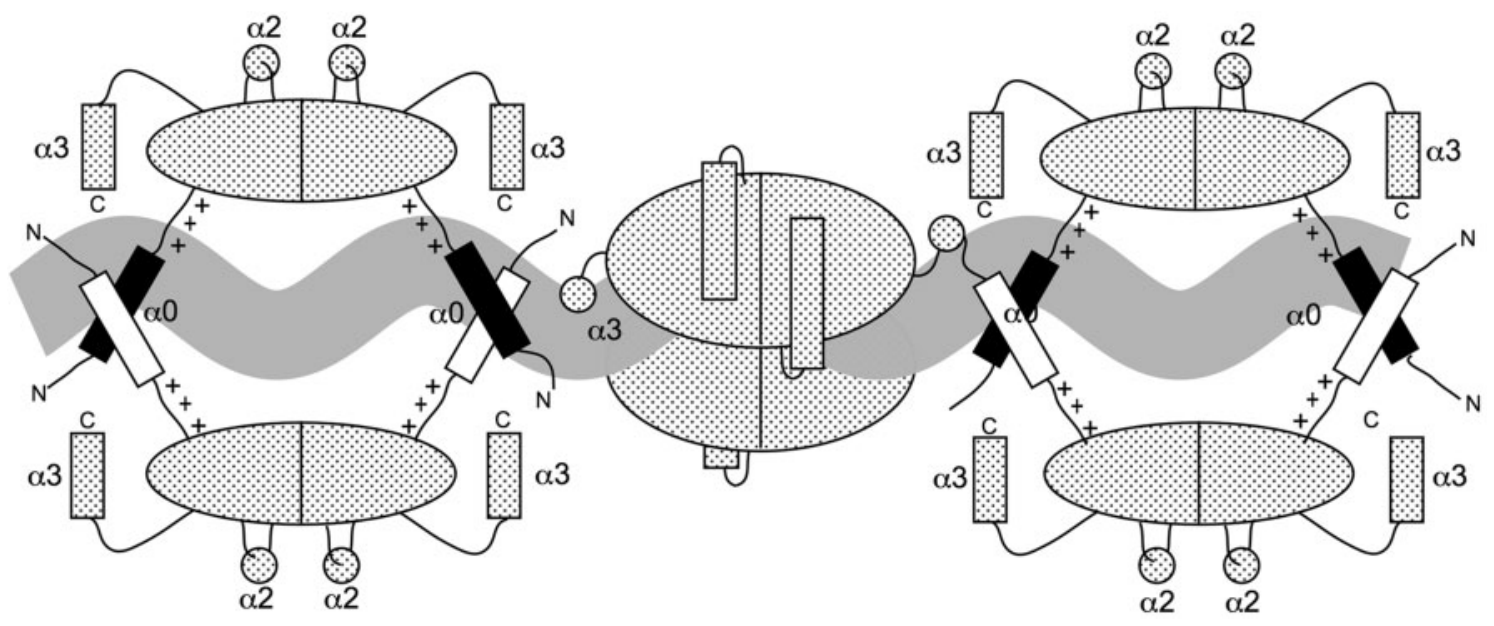

Fig. 6. Model for PRRSV nucleocapsid structure. The $\beta$-sheet domain of the $\mathrm{N}$ protein dimer is represented schematically by the dotted, oval shapes, and is flanked by $\alpha$-helices $\alpha 2$ and $\alpha 3$ (circles and rectangles, respectively). The $\mathrm{N}$ - and $\mathrm{C}$-termini are indicated, and the numbering of secondary structure elements follows that of Doan \& Dokland (2003). The predicted $\alpha$-helices $(\alpha 0)$ in the N-terminal RNA-binding domain are shown as black and white rectangles. The grey, sinusoidal shape in the background represents the viral RNA, which is assumed to interact with the positively charged residues in the $\mathrm{N}$-terminal domain (indicated by '+'). Two $\mathrm{N}$ protein dimers would constitute the ring-like shapes seen in the tomograms. 
value, each $10 \mathrm{~nm}$ link in the model would contain $43 \mathrm{nt}$ and consist of four copies of $\mathrm{N}$. This would yield an estimated 1433 copies of $\mathrm{N}$ in the core, which is in good agreement with the volume measurements.

The model we propose is not unlike others suggested for coronaviruses. Although it is not known exactly how the coronavirus $\mathrm{N}$ protein is organized into a helical nucleocapsid (Davies et al., 1981; Sturman et al., 1980), several possible models have been proposed (Chen et al., 2007; Fan et al., 2005; Jayaram et al., 2006). In some of these models, the RNA forms the centre of a helix formed by the $\mathrm{N}$ proteins, while others have proposed that the RNA is wound around the outside of a helical N protein core. However, these models are based on high-resolution structures of nucleocapsid proteins that lack most of the RNA-binding domain and do not contain RNA. The present structure of PRRSV, albeit at low resolution, pictures the core in its native state in the virus, including the complete protein-RNA complex. It is also noteworthy that the $\mathrm{N}$ protein $\mathrm{C}$-terminal domain dimers were organized end-to-end in a helical arrangement in the crystals (Doan \& Dokland, 2003).

Experiments are under way to reconstitute the core filaments in vitro from overexpressed $\mathrm{N}$ and RNA. We previously showed that refolding of $\mathrm{N}$ in the presence of tRNA gave rise to filamentous structures (Doan \& Dokland, 2003). A reverse genetics system might also allow specific proteins within the virus to be tagged or labelled with gold and would be highly beneficial to these studies. However, recombinant virus yields have been too low for structural analysis. Structural information on arterivirus will hopefully lead to a better understanding of the PRRSV assembly pathway as well as the immunological properties of PRRSV that have made this pathogen such a problem to agriculture worldwide.

\section{ACKNOWLEDGEMENTS}

The authors acknowledge funding from the United States Department of Agriculture grant USDA 2006-01616 to T. D. and support from the South Dakota Animal Disease Research and Diagnostic Laboratory and South Dakota Agricultural Experiment Station to E. N.

\section{REFERENCES}

Batista, L., Pijoan, C., Dee, S., Olin, M., Molitor, T., Joo, H. S., Xiao, Z. \& Murtaugh, M. (2004). Virological and immunological responses to porcine reproductive and respiratory syndrome virus in a large population of gilts. Can J Vet Res 68, 267-273.

Benfield, D. A., Nelson, E., Collins, J. E., Harris, L., Goyal, S. M., Robison, D., Christianson, W. T., Morrison, R. B., Gorcyca, D. \& Chladek, D. (1992). Characterization of swine infertility and respiratory syndrome (SIRS) virus (isolate VR-2332). J Vet Diagn Invest 4, 127-133.

Benjamin, J., Ganser-Pornillos, B. K., Tivol, W. F., Sundquist, W. I. \& Jensen, G. J. (2005). Three-dimensional structure of HIV-1 virus-like particles by electron cryotomography. J Mol Biol 346, 577-588.
Blaha, T. (2000). The "colorful" epidemiology of PRRS. Vet Res 31, 77-83.

Brinton, M. A. (1994). Lactate dehydrogenase-elevating, equine arteritis and Lelystad viruses. In Encyclopedia of Virology, pp. 763771. Edited by R. G. Webster \& A. Granoff. London: Academic Press.

Cavanagh, D. (1997). Nidovirales: a new order comprising Coronaviridae and Arteriviridae. Arch Virol 142, 629-633.

Chang, C. K., Sue, S. C., Yu, T. H., Hsieh, C. M., Tsai, C. K., Chiang, Y. C., Lee, S. J., Hsiao, H. H., Wu, W. J. \& other authors (2005). The dimer interface of the SARS coronavirus nucleocapsid protein adapts a porcine respiratory and reproductive syndrome virus-like structure. FEBS Lett 579, 5563-5568.

Chang, J. T., Schmid, M. F., Rixon, F. J. \& Chiu, W. (2007). Electron cryotomography reveals the portal in the herpesvirus capsid. J Virol 81, 2065-2068.

Chen, C.-Y., Chang, C.-K., Chang, Y.-W., Sue, S.-C., Bai, H.-I., Riang, L., Hsiao, C.-D. \& Huang, T.-H. (2007). Structure of the SARS coronavirus nucleocapsid protein RNA-binding dimerization domain suggests a mechanism for helical packaging of viral RNA. J Mol Biol 368, 1075-1086.

Crowther, R. A., DeRosier, D. J. \& Klug, A. (1970). The reconstruction of a three-dimensional structure from projections and its application to electron microscopy. Proc R Soc Lond A Math Phys Sci 317, 319340.

Davies, H. A., Dourmashkin, R. R. \& Macnaughton, M. R. (1981). Ribonucleoprotein of avian infectious bronchitis virus. J Gen Virol 53, 67-74.

Dea, S., Sawyer, N., Alain, R. \& Athanassious, R. (1995). Ultrastructural characteristics and morphogenesis of porcine reproductive and respiratory syndrome virus propagated in the highly permissive MARC-145 cell clone. In Corona- and Related Viruses: Current Concepts in Molecular Biology and Pathogenesis, pp. 95-98. Edited by P. J. Talbot \& G. A. Levy. New York: Plenum Press.

Dea, S., Gagnon, C. A., Mardassi, H., Pirzadeh, B. \& Rogan, D. (2000). Current knowledge on the structural proteins of porcine reproductive and respiratory syndrome (PRRS) virus: comparison of the North American and European isolates. Arch Virol 145, 659-688.

Deshpande, A., Wang, S., Walsh, M. A. \& Dokland, T. (2007). Structure of the equine arteritis virus nucleocapsid protein reveals a dimer-dimer arrangement. Acta Crystallogr D Biol Crystallogr 63, 581586.

Doan, D. N. \& Dokland, T. (2003). Structure of the nucleocapsid protein of porcine reproductive and respiratory syndrome virus. Structure 11, 1445-1451.

Dokland, T. \& Lee, M. N. M. (2006). Transmission electron microscopy of biological specimens. In Techniques in Microscopy for Biomedical Applications, pp. 153-212. Edited by T. Dokland, D. W. Hutmacher, M. L. Ng \& J.-T. Schantz. Singapore: World Scientific Press.

Drygin, Y. F., Bordunova, O. A., Gallyamov, M. O. \& Yaminsky, I. V. (1998). Atomic force microscopy examination of tobacco mosaic virus and virion RNA. FEBS Lett 425, 217-221.

Fan, H., Ooi, A., Tan, Y. W., Wang, S., Fang, S., Liu, D. X. \& Lescar, J. (2005). The nucleocapsid protein of coronavirus infectious bronchitis virus: crystal structure of its $\mathrm{N}$-terminal domain and multimerization properties. Structure 13, 1859-1868.

Fang, Y., Schneider, P., Zhang, W. P., Faaberg, K. S., Nelson, E. A. \& Rowland, R. R. R. (2007). Diversity and evolution of a newly emerged North American type 1 porcine arterivirus: analysis of isolates collected between 1999 and 2004. Arch Virol 152, 1009-1017. 
Feng, W.-H., Laster, S. M., Tompkins, M., Brown, T., Xu, J.-S., Altier, C., Gomez, W., Benfield, D. \& McCaw, M. B. (2001). In utero infection by porcine reproductive and respiratory syndrome virus is sufficient to increase susceptibility of piglets to challenge by Streptococcus suis type II. J Virol 75, 4889-4895.

Frank, J. (2006). Electron Tomography: Methods for ThreeDimensional Visualization of Structures in the Cell, 2nd edn. New York: Springer.

Grunewald, K. \& Cyrklaff, M. (2006). Structure of complex viruses and virus-infected cells by electron cryo tomography. Curr Opin Microbiol 9, 437-442.

Harris, A., Cardone, G., Winkler, D. C., Heymann, J. B., Brecher, M., White, J. M. \& Steven, A. C. (2006). Influenza virus pleiomorphy characterized by cryoelectron tomography. Proc Natl Acad Sci U S A 103, 19123-19127.

Holmes, K. V. \& Lai, M. M. C. (1996). Coronaviridae: the viruses and their replication. In Fundamental Virology, 3rd edn. Edited by B. N. Fields, D. M. Knipe \& R. M. Chanock. New York: Raven Press.

Horzinek, M. C. (1981). Non-arthropod borne togaviruses. In Experimental Virology. Edited by T. W. Tinsley \& F. Brown. London: Academic Press.

Jayaram, H., Fan, H., Bowman, B. R., Ooi, A., Jayaram, J., Collisson, E. W., Lescar, J. \& Prasad, B. V. V. (2006). X-ray structures of the $\mathrm{N}$ - and C-terminal domains of a coronavirus nucleocapsid protein: implications for nucleocapsid formation. J Virol 80, 6612-6620.

Jiang, W., Baker, M. L., Wu, Q., Bajaj, C. \& Chiu, W. (2003). Applications of a bilateral denoising filter in biological electron microscopy. J Struct Biol 144, 114-122.

Kiselyova, O. I., Yaminsky, I. V., Karger, E. M., Frolova, O. Y., Dorokhov, Y. L. \& Atabekov, J. G. (2001). Visualization by atomic force microscopy of tobacco mosaic virus movement protein-RNA complexes formed in vitro. J Gen Virol 82, 1503-1508.

Kremer, J. R., Mastronarde, D. \& McIntosh, J. R. (1996). Computer visualization of three-dimensional image data using IMOD. J Struct Biol 116, 71-76.

Lucic, V., Forster, F. \& Baumeister, W. (2005). Structural studies by electron tomography: from cells for molecules. Annu Rev Biochem 74, 833-865.

Ludtke, S. J., Baldwin, P. R. \& Chiu, W. (1999). EMAN: semiautomated software for high-resolution single-particle reconstructions. J Struct Biol 128, 82-97.

Mardassi, H., Athanassious, R., Mounir, S. \& Dea, S. (1994). Porcine reproductive and respiratory syndrome virus: morphological, biochemical and serological characteristics of Quebec isolates associated with acute and chronic outbreaks of porcine reproductive and respiratory syndrome. Can J Vet Res 58, 55-64.

Mardassi, H., Massie, B. \& Dea, S. (1996). Intracellular synthesis, processing and transport of proteins encoded by ORFs 5 to 7 of porcine reproductive and respiratory syndrome virus. Virology 221, 98-112.

McEwen, B. F., Downing, K. H. \& Glaeser, R. M. (1995). The relevance of dose-fractionation in tomography of radiation-sensitive specimens. Ultramicroscopy 60, 357-373.

Meng, X. J. (2000). Heterogeneity of porcine reproductive and respiratory syndrome virus: implications for current vaccine efficacy and future vaccine development. Vet Microbiol 74, 309-329.

Meng, X. J., Paul, P. S., Halbur, P. G. \& Lum, M. A. (1995). Phylogenetic analyses of the putative M (ORF6) and N (ORF7) genes of porcine reproductive and respiratory syndrome virus (PRRSV): implication for the existence of two genotypes of PRRSV in the U.S.A. and Europe. Arch Virol 140, 745-755.
Meulenberg, J. J., Petersen-den Besten, A., De Kluyverer, E. P., Moormann, R. J., Schaaper, W. M. \& Wenswoort, G. (1995). Characterization of proteins encoded by ORFs 2 to 7 of Lelystad virus. Virology 206, 155-163.

Murtaugh, M. P., Xiao, Z. \& Zuckermann, F. (2002). Immunological responses of swine to porcine reproductive and respiratory syndrome virus infection. Viral Immunol 15, 533-547.

Nelsen, C. J., Murtaugh, M. P. \& Faaberg, K. S. (1999). Porcine reproductive and respiratory syndrome virus comparison: divergent evolution on two continents. J Virol 73, 270-280.

Neuman, B. W., Adair, B. D., Yoshioka, C., Quispe, J. D., Milligan, R. A., Yeager, M. \& Buchmeier, M. J. (2006a). Ultrastructure of SARS$\mathrm{CoV}$, FIPV, and MHV revealed by electron cryomicroscopy. Adv Exp Med Biol 581, 181-185.

Neuman, B. W., Adair, B. D., Yoshioka, C., Quispe, J. D., Orca, G., Kuhn, P., Milligan, R. A., Yeager, M. \& Buchmeier, M. J. (2006b). Supramolecular architecture of severe acute respiratory syndrome coronavirus revealed by electron cryomicroscopy. J Virol 80, 79187928.

Perera, R., Owen, K. E., Tellinghuisen, T. L., Gorbalenya, A. E. \& Kuhn, R. J. (2001). Alphavirus nucleocapsid protein contains a putative coiled coil $\alpha$-helix important for core assembly. J Virol 75, $1-10$.

Pettersen, E. F., Goddard, T. D., Huang, C. C., Couch, G. S., Greenblatt, D. M., Meng, E. C. \& Ferrin, T. E. (2004). UCSF Chimera a visualization system for exploratory research and analysis. J Comput Chem 25, 1605-1612.

Plagemann, P. G. W. (1996). Lactate dehydrogenase-elevating virus and related viruses. In Fields Virology, 3rd edn, pp. 1105-1120. Edited by B. N. Fields, D. M. Knipe \& P. M. Howley. Philadelphia: Lippincott-Raven Publishers.

Rossow, K. D. (1998). Porcine reproductive and respiratory syndrome. Vet Pathol 35, 1-20.

Snijder, E. J. \& Meulenberg, J. J. M. (1998). The molecular biology of arteriviruses. J Gen Virol 79, 961-979.

Snijder, E. J., Horzinek, M. C. \& Spaan, W. J. M. (1993). The coronaviruslike superfamily. Adv Exp Med Biol 342, 235-244.

Sturman, L. S., Holmes, K. V. \& Behnke, J. (1980). Isolation of coronavirus envelope glycoproteins and interaction with the viral nucleocapsid. J Virol 33, 449-462.

Wissink, E. H. J., Kroese, M. V., Maneschijn-Bonsing, J. G., Meulenberg, J. J. M., van Rijn, P. A., Rijsewijk, F. A. M. \& Rottier, P. J. M. (2004). Significance of the oligosaccharides of the porcine reproductive and respiratory syndrome virus glycoproteins GP2a and GP5 for infectious virus production. J Gen Virol 85, 3715-3723.

Wissink, E. H. J., Kroese, M. V., van Wijk, H. A. R., Rijsewijk, F. A. M., Meulenberg, J. J. \& Rottier, P. J. M. (2005). Envelope protein requirements for the assembly of infectious virions or porcine reproductive and respiratory syndrome virus. J Virol 79, 1249512506.

Wootton, S. K. \& Yoo, D. (2003). Homo-oligomerization of the porcine reproductive and respiratory syndrome virus nucleocapsid protein and the role of disulfide linkages. J Virol 77, 4546-4557.

Wright, E. R., Schooler, J. B., Ding, H. J., Kieffer, C., Fillmore, C., Sundquist, W. I. \& Jensen, G. J. (2007). Electron tomography of immature HIV-1 virions reveals the structure of the CA and SP1 Gag shells. EMBO J 26, 2218-2216.

Wu, W. H., Fang, Y., Farwell, R., Steffen-Bien, M., Rowland, R. R., Christopher-Hennings, J. \& Nelson, E. A. (2001). A 10-kDa structural protein of porcine reproductive and respiratory syndrome virus encoded by ORF2b. Virology 287, 183-191. 
Yoo, D., Wootton, S., Li, G., Song, S. \& Rowland, R. R. (2003). Colocalization and interaction of the porcine arterivirus nucleocapsid protein with the small nucleolar RNA-associated protein fibrillarin. J Virol 77, 12173-12183.

Yu, I. M., Oldham, M. L., Zhang, J. \& Chen, J. (2006). Crystal structure of the severe acute respiratory syndrome (SARS) coronavirus nucleocapsid protein dimerization domain reveals evolutionary linkage between Corona- and Arteriviridae. J Biol Chem 281, 1713417139 .

Zimmerman, J. J., Yoon, K. J., Wills, R. W. \& Swenson, S. L. (1997). General overview of PRRSV: a perspective from the United States. Vet Microbiol 55, 187-196. 